\title{
Über die verschiedenen Arten der linealen Erzeugung algebraischer Oberflächen.
}

(Von dem Herrn Prof. H. Gra/smann, Oberlehrer am Gymnasio zu Stettin.)

Die drei Gattungen räumlicher Elemente, nämlich die Puncte, oder die Elemente erster Stufe; die geraden Linien, oder die Elemente zweiter Stufe; die Ebenen, oder die Elemente dritter Stufe, gestatten sechs verschiedene Combinationen zu zweien, welche sich, je nachdem die Summe der Stufenzahlen kleiner als 4 , oder gröfser als 4 , oder gleich 4 ist, in drei Gruppen sondern lassen. Für jede dieser sechs Combinationen habe ich in der vorhergehenden Abhandlung den Begriff des stereometrischen Products beider Elemente bestimmt. Wenn die Summe der Stufenzahlen kleiner als 4 war, so war das stereometrische Product die Verbindung beider Elemente (Verbindungslinie und Verbindungs-Ebene), wenn grö/ser als 4, ihr Durchschnitt. Beide Bestimmungen ergaben sich, als zu einander reciprok. Wenn die Summe der Stufenzahlen gleich 4 war, so setzten wir als das Product der Elemente eine Größse, welche, einem Elemente als Factor beigefügt, die Lage des Elements unverändert läfst. Wir nannten eine solche Gröfse eine Gröfse nullter Stufe. In diesem Sinne ergab sich, dafs die Stufe eines beliebig zusammengesetzten Products stets der Summe der Stufenzahlen seiner sämmtlichen Factoren congruent ist, in Bezug auf den Modul 4. Alle 6 Arten der Producte wurden gleich Null gesetzt, wenn die Elemente, welche die Factoren des Products bildeten, vereinigt lagen. Congruent hingegen nannten wir zwei Elemente, wenn sie (als unendlich angenommen) sich gegenseitig deckten; zwei Gröfsen nullter Stufe aber nannten wir congruent, wenn sie entweder beide Null, oder beide ungleich Null waren. Wir untersuchten dann, in wie weit man die Form eines Products verändern könne, so dafs das ursprüngliche und das neue Product einander congruent seien. Es ergaben sich dabei besonders folgende Formeln und Sätze, in welchen $\mathbf{A}, \mathbf{B}, \Gamma, \mathbf{A}_{n}$, $\mathfrak{P}$ Elemente darstellen, deren Stufenzahlen beziehlich $\mathfrak{a}, \mathfrak{b}, \mathfrak{c}, \mathfrak{a}_{n}, 0$ sein mögen: 


$$
\begin{gathered}
\text { 1. } \mathrm{AB} \equiv \mathrm{BA}, \\
2 . \quad \mathrm{AB} \Gamma \equiv \mathrm{A} \Gamma \mathrm{B} \equiv \mathrm{A}(\mathrm{B} \Gamma) \text { etc. } \\
\text { wenn } \mathfrak{a}+\mathfrak{b}+\mathfrak{c}<5, \text { oder }>7 \text { ist. } \\
\text { 3. } \quad \mathrm{ABIB} \equiv \mathrm{AB}(\Gamma \mathrm{B}) \equiv \mathrm{A}(\Gamma \mathrm{B}) \mathrm{B}, \\
\text { 4. } \quad \mathrm{A}_{1} \mathrm{~A}_{2} \ldots \mathrm{A}_{n} \equiv \mathrm{A}_{n} \ldots \mathrm{A}_{2} \mathrm{~A}_{1} \equiv \mathrm{A}_{1}\left(\mathrm{~A}_{n} \ldots \mathrm{A}_{2}\right), \\
\text { wenn } \mathfrak{a}_{1}+\mathfrak{a}_{2}+\ldots+\mathfrak{a}_{n} \equiv 0(\bmod 4) \text { ist. }
\end{gathered}
$$

5. $\mathfrak{P}=0$ ist die Gleichung einer Oberfläche nter Ordnung, wenn $\mathfrak{Y}$ ein Product nullter Stufe ist, in welchem der die Oberfläche construirende Punct $\boldsymbol{n}$ mal als Factor vorkommt.

6. Wenn ein Product nullter Stufe $\mathfrak{P}$ noch einen Factor nullter Stufe $\mathfrak{D}$. enthält, und $\Re$ das Product bezeichnet, welches aus $\mathfrak{P}$ übrig bleibt, wenn man darin $\mathfrak{S}$ wegläfst; so ist

$$
\mathfrak{P} \equiv \mathfrak{D}
$$

und die Gleichung

$$
\mathfrak{S}=\mathfrak{Q} \Re=0 \text { zerfällt in } \mathfrak{D}=0 \text { und } \Re=0 .
$$

Der Zweck des gegenwärtigen Aufsatzes ist, den allgemeinen Satz über die lineale Erzeugung der Oberflächen, wie er in (5.) enthalten ist, möglichst anschaulich und für die Anwendung bequem zu gestalten und auseinander zu legen. Dabei ist vermöge (6.) nur nölhig, den Fall zu berücksichtigen, wo $\mathfrak{P}$ keinen Factor nullter Stufe mehr enthält, d. h. wo bei der fortschreitenden Bildung des Products $\mathfrak{P}$ die Summe der Stufenzahlen nicht eher durch 4 theilbar wird, als bis das ganze Product gebildet ist. Ich werde daher in den nächsten fünf Paragraphen den Fall, wo die Summe der Stufenzahlen durch 4 theilbar ist, ein für alle Mal ausschliefsen. Wie bisher, sollen die kleinen lateinischen Buchstaben (mit einziger Ausnabme des n) Puncte bezeichnen, die grofsen lateinischen Buchstaben gerade Linien, die kleinen griechischen Buchstaben Ebenen.

$$
\text { S. } 1 \text {. }
$$

Product eines beweglichen Elements, mit einem festen.

Das bewegliche Element sei $x, \xi$ oder $X$, das feste Element $a, x$ oder A. Indem ich die 6 Producte, welche, unter Ausschlufs der Stufensumme 4, aus einem der beweglichen und einem der festen Elemente sich bilden lassen, betrachte, will ich anf den Grad der Beweglichkeit des entstehenden Products und auf die entstehenden projectivischen Grundgebilde 
aufmerksam machen. Ich nenne ein Element $n$ fach, oder im nten Grade beweglich, wenn von den Zahlcoëfficienten, durch welche seine Lage bestimmt ist, $n$ auf ganz unabhängige Weise veränderlich sind. Also ist, z. B. ein Punct in einer Geraden einfach beweglich, ein Punct in einer Ebene zweifach, ein Punct im Raume dreifach. Eben so verhält es sich mit einer Ebene, welche durch eine feste Gerade, oder durch einen festen Punct gehen, oder frei im Raume beweglich sein soll. Ferner, eine Gerade, wenn sie in einer festen Ebene liegen und zugleich durch einen festen Punct derselben gehen soll, ist einfach beweglich; wenn sie in einer festen Ebene liegen, oder durch einen festen Punct gehen soll, ist sie zweifach beweglich; wenn sie eine andere Gerade schneiden soll, dreifach, und wenn sie sich frei im Raume bewegen darf, vierfach. Nimmt man nun an, $x, \xi, X$ seien frei im Ranme beweglich, so ist

1) $x a$ zweifach beweglich und stellt einen Strahlenbüschel (im Raume) dar;

2) $\xi \alpha$ ist zweifach beweglich und stellt eine liniirte Ebene dar;

3) $\boldsymbol{x A}$ ist einfach beweglich und stellt einen Ebenenbüschel (erster Stufe) dar;

4) $\xi \boldsymbol{A}$ ist einfach beweglich und stellt eine punctirte Gerade dar;

5) $\boldsymbol{X} u$ ist zweifach beweglich und stellt einen Ebenenbüschel zweiterStufe dar;

6) $\boldsymbol{X} \alpha$ ist zweifach beweglich und stellt eine punctirte Ebene dar.

Hierzu füge ich, der Übersicht wegen, noch die folgenden beiden Producte dreier Factoren:

7) $x A \alpha$, sind einfach beweglich und stellen ebene Strahlenbüschel dar, und zwar 7) als Durchschnitt eines Ebenenbüschels und einer Ebene 8) als Verbindung einer punctirten Geraden mit einem Puncte.

Diese projectivischen Grundgebilde sind paarweise zu einander reciprok. Ich werde diejenigen derselben, deren Element einfach oder zweifach beweglich ist, beziehlich, Gebilde erster oder zweiter Stufe nennen; wodurch die Benennung des fünften Gebildes gerechtfertigt scheint.

Um die Nothwendigkeit dieser Unterscheidung in projectivische Gebilde erster und zweiter Stufe nachzuweisen, führe ich gelegentlich folgenden, leicht zu erweịsenden Satz an: Bei zwei Gebilden derselben Art, welche zu einander projectivisch sein sollen, kann man, je nachdem sie von der ersten oder zweiten Stufe sind, 3 oder 4 beliebige Elemente des einen Gebildes, mit eben so vielen der andern als entsprechend setzen; aber dann ist zu jedem Elemente des einen Gebildes das entsprechende des andern bestimmt; vorausgesetzt jedoch, 
dafs im zweiten Falle von den gewählten 4 Elementen desselben Gebildes keine 3 einem und demselben Gebilde erster Stufe angehören.

Es ist bei der Auffassung dieser projectivischen Gebilde wichtig, festzuhalten, dafs die punctirte Ebene und die liniirte Ebene, und eben so der Ebenenbüschel zweiter Stufe und der räumliche Strahlenbüschel, nur in Bezug auf die Gattung der Elemente sich unterscheiden; so dafs nämlich zwei projectivische punctirte Ebenen zugleich projectivisch sind in Bezug auf die Verbindungslinien ihrer entsprechenden Punctenpaare, und zwei projectivische Ebenenbüschel zugleich in Bezug auf die Durchschnittsstrahlen der entsprechenden Ebenenpaare; und umgekehrt. Wo es auf die Unterscheidung der ursprünglichen Elemente nicht ankommt, werde ich, mit Steiner, die ersteren Gebilde, ohne Weiteres, Ebenen, die lelzteren räumliche Strahlenbüschel nennen.

\$. 2 .

Fortschreitende Mulliplication eines frei beweglichen Punctes $x$, mit einer Reihe fester Elemente.

Es lassen sich zwei Hauptfälle' unterscheiden, je nachdem das entstehende Product einfach, oder zweifach beweglich ist. Es werde der letzte Fall zuerst betrachtet. Der Punct $x$ kann in diesem Falle nur mit einem Puncte multiplicirt werden, da das Product mit einer Geraden einfach beweglich ist. Es sei dieser Punct $a$. Das Product $x a$ ist nur dann Null, wenn $x$ in $a$ fällt; in jedem andern Falle liefert $x a$ eine Gerade. Diese Gerade könnte nun mit einem Puncte $b$, oder einer Ebene $\alpha$, multiplicirt werden; allein $x a b$ kann zugleich als Product von $x$ mit der Geraden $a b$ betrachtet werden, und würde daher einfach beweglich sein. Es ergiebt sich also das Product $x u \alpha$. Wenn $a$ in $\alpha$ liegt, so lassen sich nach Formel (3.) $a$ und $\alpha$ vertauschen, und man erhält $x \alpha . a$; was in einen variablen Factor nullter Stufe und einen festen Punct zerfällt. Da wir auch diesen Fall ausschlossen, so bleibt nur übrig, dafs $a$ aufserhalb $\alpha$ liegl; wo dann $x a . \alpha$ eine punctirte Ebene darstellt. Der Punct $x a . \alpha$ kann nun wieder mit einem Puncte $b$ multiplicirt werden. Wenn $b$ in $\alpha$ liegt, so lälst sich wiederum $b$ mit $\alpha$ vertauschen und man erhält $x a b \alpha$ oder $x(a b) \alpha$, und man kommt wieder auf den Fall der einfachen Beweglichkeit zurück. Von hier an wiederholt sich dieselbe Schlufsreihe, und man gelangt zu dem Satze:

"Wenn ein frei beweglicher Punct $x$ fortschreitend mit einer Reihe fester Elemente multiplicirt wird, so geht dann, und nur dann, ein zweifach 
bewegliches Product hervor, wenn diese Reihe mit einem Puncte beginnt, abwechselnd aus Puncten und Ebenen besteht, und dabei jeder Punct aufserhalb der ihm vorhergehenden und der ihm nachfolgenden Ebene liegt. Das Product wird in diesem Falle nur Null, wenn $x$ mit dem ersten Puncte jener Reihe zusammenfällt."

Ferner

"Wenn der Punct $x$ mit einer abwechselnden Reihe von Puncten und Ebenen multiplicirt wird, und einer dieser Puncte in die ihm nachfolgende oder vorhergehende Ebene fäll, so kann man diese beiden Factoren vertauschen, und erhält im ersten Falle einen variablen Factor nullter Stufe, der mit einem festen Element multiplicirt ist, im letzten Falle ein einfach bewegliches Product, indem an die Stelle des Puncts eine Gerade tritt."

\section{§. 3 .}

Fortsetzung.

Es werde jetzt der Fall der einfachen Beweglichkeit betrachtet. Am einfachsten ist es hier, $x$ fortschreitend mit einer Reihe von Geraden $\boldsymbol{A}, \boldsymbol{B}, \ldots$ zu multipliciren. Das Product $\boldsymbol{x A}$ wird Null, wenn $\boldsymbol{x}$ in $\boldsymbol{A}$ fält. Dies ausgeschlossen, stellt $\boldsymbol{x} \boldsymbol{A}$ einen Ebenenhüschel dar. Wenn nun von den folgenden Geraden keine die vorhergehende schneidet (der unendlich entfernte Durchschnittspunct immer als solcher mit gerechnet), so stellt das Product abwechselnd Ebenenbüschel und punctirte Geraden dar. Ist nun das Product, bis zu irgend einem Factor hin, $\equiv p$, und es schneiden sich die beiden folgenden festen Geraden $\boldsymbol{A}$ und $\boldsymbol{B}$, so sei dieser Punct $\boldsymbol{c}$, und die Geraden seien $a c$ und $b c$. Dann erbält man $\boldsymbol{p A B} \equiv \boldsymbol{p a c}(b c) \equiv p a c b . c$ [nach Formel 3]; d. h. es zerfällt das Product in einen variablen Factor nullter Stufe und einen festen Punct. Das Entsprechende geschieht im reciproken Falle. Also ergiebt sich folgender Satz:

„Wenn ein frei beweglicher Punct mit einer Reibe fester Geraden multiplicirt wird, so ist das Product dann, und nur dann, einfach beweglich, wenn keine dieser Geraden die folgende schneidet; und das Product wird in diesem Falle nur dann Null, wenn $x$ in die erste Gerade jener Reihe fällt. Wenn jedoch eine der Geraden die folgende schneidet, so kann man statt dieser beiden Geraden eine Ebene und einen Punct setzen; und zwar die Ebene in der sie liegen und den Punct in dem sie sich Crelle's Journal f. d. M. Bd. XLIX. Heft 1. 
schneiden, und es zerfällt dadurch das Product in einen variablen Factor nullter Stufe und in ein festes Element."

Ich werde jetzt zeigen, dafs man jeden Fall, in welchem ein beweglicher Punct $x$, mit einer Reihe fester Elemente multiplicirt, ein einfach bewegliches Element, und zwar einen Punct oder eine Ebene giebt, auf den so eben betrachteten Fall zurückführen kann.

Es sei zuerst $p$ ein zweifach bewegliches Product, und eben so pac; dann trete noch die Gerade $\boldsymbol{B}$ hinzu. Es sei $\boldsymbol{b}$ der Punct, in welchem die Gerade $\boldsymbol{B}$ die Ebene $\alpha$ schneidet, und $\alpha \equiv \boldsymbol{A} b, \boldsymbol{B} \equiv \boldsymbol{b c}$, so ist:

$$
p a \alpha B \equiv p a(A b)(b c) \equiv p a(A b) b c \text { [nach Formel 2]. }
$$

Aber

$$
\begin{gathered}
p a(A b) b \equiv a b A b \text { [nach Formel 3], also } \\
p a \alpha B \equiv p a b A b c \equiv p(a b) \boldsymbol{A}(b c) \text { [nach Formel 2]. }
\end{gathered}
$$

Man hat also statt des Puncts $a$ und der Ebene $\alpha$ gerade Linien erhalten. Nämlich, wenn man den Durchschnittspunct von $B$ und $\alpha$ durch $b$ bezeichnet, so kann man statt des Punctes $a$ die Gerade $a b$ und statt der Ebene $\alpha$ eine beliebige Gerade dieser Ebene setzen, die jedoch nicht durch den Punct $b$ gehen darf. Durch wiederholte Anwendung dieses Verfahrens gelangt man zu folgendem Satze:

„Wenn ein beweglicher Punct mit einer Reihe abwechselnder fester Puncte und Ebenen multiplicirt wird und auf die letzte Ebene eine feste Gerade folgt, so kann man statt aller fester Puncte und Ebenen Gerade setzen. Nämlich wenn man das ganze Product umkehrt und das umgekehrte Product nach und nach construirt, so erhält man eine Reihe von Hülfspuncten, die in den festen Ebenen liegen, und eine Reihe von Geraden, die durch die festen Puncte gehen. Diese Geraden kann man statt der festen Puncte setzen, und statt jeder Ebene eine Gerade, die in dieser Ebene liegt, aber nicht durch den in der Ebene liegenden Hülfspunct gebt."

In Formeln ausgedrückt, würde der Satz:

$$
x a_{1} \alpha_{1} a_{1} \alpha_{2} \ldots a_{n} \alpha_{n} B \equiv x\left(a_{1} c_{1}\right) A_{1}\left(c_{1} c_{2}\right) A_{2} \ldots\left(c_{n-1} c_{n}\right) A_{n} B
$$

lauten, wenn;

$$
\begin{aligned}
B \alpha_{n} & \equiv c_{n}, c_{n} a_{n} \alpha_{n-1} \equiv c_{n-1}, \ldots c_{2} a_{2} \alpha_{1} \equiv c_{1} \\
\alpha_{n} & \equiv c_{n} A_{n}, \quad a_{n-1} \equiv c_{n-1} A_{n-1}, \ldots \alpha_{1} \equiv c_{1} A_{1}
\end{aligned}
$$


ist. Noch will ich bemerken, dafs sich auch die gefundene Form des Products noch auf die Form $x\left(a_{1} c_{1}\right) \boldsymbol{B}_{1}\left(c_{2} c_{3}\right) \boldsymbol{B}_{3} \ldots$ reduciren läfst, wo $\boldsymbol{B}$, die Durchschnittslinie der Ebenen $\alpha_{1}$ und $\alpha_{2}$ (oder $c_{1} A_{1}$ und $c_{2} A_{2}$ ), $B_{3}$ die der Ebenen $\alpha_{3}$ und $\alpha_{4}$ ist; u. s. w.

Es bleibt jetzt nur noch zu zeigen, dafs sich jedes Product von $x$ mit einer Elementenreihe, die mit einer Geraden beginnt, auch, falls das Product wieder ein Punct oder eine Ebene sein soll, stets auf das Product von $\boldsymbol{x}$ mit einer Reihe von lauter Geraden zurückführen läfst. Durch die Multiplication mit einer Reihe fester Geraden geht entweder ein Punct $\boldsymbol{p}$, oder eine Ebene hervor. Da beide Fälle zu einander reciprok sind, so braucht man nur den einen zu betrachten. Wir nehmen an, der Punct $p$ sei entweder $\equiv x$, oder er sei durch Multiplication von $x$ mit einer Reihe von festen Geraden entstanden. Es trete noch eine Gerade $\boldsymbol{A}$ hinzu, so ist $\boldsymbol{p} \boldsymbol{A}$ eine Ebene. Soll nun zu dieser Ebene ein Element, welches keine Gerade ist, hinzutreten, so kann dieses Element (da die Stufensumme 4 ausgeschlossen ist) nur eine Ebene $\alpha$ sein. Nun ist $\boldsymbol{p A} \alpha$ eine Gerade. Diese kann mit einer Ebene $\beta$, oder mit einem Punct $b$ zusammentreten: aber $p A \alpha \beta$ ist nach Formel (2) $\equiv \boldsymbol{p} \boldsymbol{A}(\alpha \beta)$, also wäre $\boldsymbol{p A}$ wieder mit einer Geraden $(\alpha \beta)$ multiplicirt; gegen die Annahme. Es bleibt daher nur übrig, das Product $\boldsymbol{p A \alpha b}$ zu betrachten. Es sei $c$ der Durchschnitt von $A$ und $\alpha$, und $A \equiv a c, \alpha \equiv c B$, so ist $\boldsymbol{p A} \alpha \boldsymbol{b} \equiv \boldsymbol{p}(\boldsymbol{a c})(\boldsymbol{c B}) \boldsymbol{b} \equiv \boldsymbol{p a c}(\boldsymbol{c B}) \boldsymbol{b}$ [nach F. 2] $\equiv \boldsymbol{p a c} \boldsymbol{B c b}$ [nach F. 3] $\equiv \boldsymbol{p}(\boldsymbol{a c}) \boldsymbol{B}(\boldsymbol{c} b)$. Also ist auch dies Product auf ein Product mit lauter festen Geraden reducirt. Wir haben demnach folgenden Satz erlangt:

„Jedes Product eines frei beweglichen Punctes $x$ mit einer Reihe fester Elemente läfst sich, wenn das Product ein einfach bewegliches Element, und zwar ein Punct oder eine Ebene ist, in der Form darstellen, dafs alle festen Elemente Gerade sind."

Fasset man die gefundenen Sätze mit den reciproken Sätzen zusammen, so erhält man folgenden allgemeinen Satz:

„Wenn ein frei bewegliches Element, und zwar ein Punct, oder eine Ebene, mit einer Reihe fester Elemente multiplicint und das Product wieder ein bewegliches Element, und zwar ein Punct oder eine Ebene ist, so kann man statt der Reihe der festen Elemente entweder eino Reihe abwechselnder festen Puncte und Ebenen, oder eine Reibe fester Geraden setzen, je nachdem das Product ein zweifach oder einfach bewegliches Element ist; und zwar haben beide Reihen nothwendig die 
Eigenschaft, dafs keine zwei aufeinander folgenden Elemente vereinigt liegen. Findet hingegen diese vereinigte Lage Statt, so nimmt der Grad der Beweglichkeit mindestens um 1 ab."

Dieser Satz reicht für die Multiplication eines beweglichen Elements mit einer Reihe fester, vollkommen aus. Denn ist das bewegliche Element eine Gerade, so mufs diese entweder mit einer festen Ebene, oder mit einem festen Puncte in Combination treten, und giebt dann einen zweifach beweglichen Punct, oder eine zweifach bewegliche Ebene; und für diese wurde die weitere Combination mit festen Elementen bereits betrachtet. Und ist das Product eine Gerade, so kann dieselbe nur durch Multiplication zweier Puncte oder zweier Ebenen entstanden sein; und für beide wurden die Gesetze aufgestellt.

\section{S. 4.}

Lineale Bewegung offener Figuren.

Es sei zunächst die Aufgabe, das bewegliche Product eines beweglichen Elements mit einer Reihe fester Elemente für den Fall zu construiren, dafs sowohl das bewegliche Element, als das Product, ein Punct, oder eine Ebene ist. In diesem Falle kann man nach (\$.2.) statt der Reihe der festen Elemente, entweder eine Reihe abwechselnder Puncte und Ebenen, oder eine Reihe von Geraden einführen; und zwar so, dafs keine zwei aufeinander folgende Elemente dieser Reihen vereinigt liegen. Ich werde mich für die Darstellung dieser Construction des Begriffs der offenen Figur bedienen. Darunter verstehe ich (S. dieses Journal Bd. 36. S. 5) eine Reihe von Puncten und Geraden, in welcher auf jeden Punct eine durch ihn gehende Gerade, und auf jede Gerade ein in ihr liegender Punct folgt, gleich viel ob diese Geraden oder Puncte in derselben Ebene liegen, oder nicht. Das AnfangsElement der Reihe kann, eben so wie das End-Element derselben, ein Punct, oder eine Gerade sein. Alle Zwischen-Elemente der Reihe (d. h. welche nicht Grenz-Elemente derselben sind) nenne ich Seiten oder Ecken der offenen Figur, je nachdem sie Gerade, oder Puncte sind. Das Product eines beweglichen Punctes $x$ mit einer abwechselnden Reihe von Puncten und Ebenen $a_{1}, \alpha_{1}, \ldots a_{n}, \alpha_{n}$ betrachtet, deren keine zwei aufeinander folgende vereinigt liegen), ist Null, wenn $x$ in $a_{1}$ liegt; in jedem andern Falle ist das Product die letzte Ecke einer offenen Figur, deren Angriffspunct $x$ ist, deren Seiten durch die festen Puncte $a_{1} \ldots a_{n}$ gehen und deren Ecken in den festen Ebenen $\alpha_{1} \ldots \alpha_{n}$ liegen. Betrachtet man, zweitens, das Product eines beweg- 
lichen Punctes $x$ mit einer geraden Anzahl gerader Linien $\boldsymbol{A}_{1}, \boldsymbol{B}_{1}, \ldots \boldsymbol{A}_{n}, \boldsymbol{B}_{n}$, (deren keine zwei aufeinander folgende sich schneiden), so zeigt sich dasselbe $=$ Null, wenn $x$ in der Geraden $A_{1}$ liegt; in jedem andern Falle ist das Product die letzte Ecke einer offenen Figur, deren Anfangspunct $x$ ist, deren Seiten durch die Geraden $\boldsymbol{A}_{1}, \ldots \boldsymbol{A}_{n}$ gehen und deren Ecken in der Geraden $\boldsymbol{B}_{1}, \ldots \boldsymbol{B}_{n}$ liegen. Ferner werde das Product von $\boldsymbol{x}$ mit einer ungeraden Anzahl gerader Linien $\boldsymbol{A}_{1}, \boldsymbol{B}_{1}, \ldots \boldsymbol{A}_{n}, \boldsymbol{B}_{n}, \boldsymbol{A}_{n+1}$ betrachtet, (deren keine zwei aufeinanderfolgende sich schneiden). Legt man nun, vorausgesetzt, dafs $\boldsymbol{x}$ nicht in $\boldsymbol{A}_{1}$ liegt, in welchem Falle das Product Null ist, eine offene Figur hindurch, deren Anfangspunct $\boldsymbol{x}$ ist, deren Seiten durch die Geraden $\boldsymbol{A}_{1}, \ldots \boldsymbol{A}_{n+1}$ gehen und deren Ecken in den Geraden $\boldsymbol{B}_{1}, \ldots \boldsymbol{B}_{n}$ liegen, so ist durch eine bestimmte Lage des Anfangspuncts $x$ zwar die letzte Ecke in $\boldsymbol{B}_{n}$ bestimmt, aber nicht die letzte Seite, die durch $\boldsymbol{A}_{n+1}$ geht; vielmehr ist der geometrische Ort derselben eine Ebene, und diese Ebene ist eben jenem Producte congruent.

Es bleiben nur noch die reciproken Fälle zu betrachten, wo statt des Punctes $x$ eine Ebene $\xi$ eintritt. Man könnte hier der offenen Figur ihr reciprokes Gebilde substituiren; doch ist es in vielen Fällen vortheilhaft, auch hier die offene Figur zu Grunde zu legen. Hat man das Product $\xi \alpha_{1} a_{1} \ldots \alpha_{n} a_{n}$ zu construiren, so läfst sich eine offene Figur zu Grunde legen, deren Anfangsstrahl in der Ebene $\xi$ liegt, deren Ecken in den Ebenen $\alpha_{1} \ldots \alpha_{n}$ liegen und deren Seiten durch die Puncte $a_{1}, \ldots a_{n}$ gehen. Wenn die Ebene $\xi$ fest ist (ohne mit $\alpha_{1}$ zusammenzufallen), so ist dennoch die ganze offene Figur beweglich, und der geometrische Ort ihrer letzten Seite ist eine Ebene, welche dem obigen Producte congruent ist. Ferner ist das Product $\boldsymbol{\xi} \boldsymbol{A}_{1} \boldsymbol{B}_{1} \ldots \boldsymbol{A}_{n} \boldsymbol{B}_{n}$ der geometrische Ort der letzten Seite einer offenen Figur, deren Anfangsstrabl in der Ebene $\xi$ liegt, deren Ecken in den Geraden $A_{1} \ldots A_{n}$ liegen, und deren Seiten durch die Geraden $B_{1} \ldots B_{n}$ gehen. Endlich, das Product $\xi A_{1} B_{1} \ldots A_{n} B_{n} A_{n+1}$ ist der letzten Ecke einer Figur congruent, deren Anfangsstrahl in der Ebene $\xi$ liegt, deren Ecken in den Seiten $\boldsymbol{A}_{1} \ldots \boldsymbol{A}_{n+1}$ liegen und deren Seiten durch die Geraden $\boldsymbol{B}_{1} \ldots \boldsymbol{B}_{n}$ gehen.

Ich werde alle diese sechs Bewegungen der offenen Figuren lineale nennen. Es giebt also zwei Arten der linealen Bewegung offener Figuren, deren erste darin besteht, dafs sich alle Ecken und Seiten in festen geraden Linien bewegen, die andere darin, dafs sich alle Ecken in festen Ebenen bewegen, während alle Seiten durch feste Puncte gehen. Bei beiden Bewegungen soll 
wieder der Fall ausgeschlossen bleiben, wo irgend zwei feste Elemente, in denen sich zwei aufeinander folgende Elemente der offenen Figur bewegen sollen, vereinigt liegen. Im ersteren Falle sind alle Ecken der offenen Figur einfach beweglich, im letzteren zweifach; daher will ich jene erstere Art der linealen Bewegung gleichfalls eine einfache, letztere eine zweifache nennen. In beiden Fällen ist jede Ecke (und eben so der geometrische Ort jeder Seite) einer lineal beweglichen offenen Figur einem Producte congruent, dessen erster Factor der geometrische Ort des Anfangs-Elements ist, und dessen folgende Factoren nach der Reihe diejenigen festen Elemente sind, welchen die Ecken und Seiten der offenen Figur, bis zu der betrachteten Ecke (oder Seite) hin, vereinigt liegen sollen.

\section{\$. 5 .}

Construction der Producte mit mehreren variablen Factoren, durch Verkettungen offener Figuren.

Wenn zwei variable Factoren zusammentreten, so sind (immer noch das Product nullter Stufe ausgeschlossen) folgende Fälle möglich: Entweder a) es treten zwei Puncte zusammen, oder b) zwei Ebenen, oder c) eine Gerade und ein Punct, oder $d$ ) eine Gerade und eine Ebene.

In den ersten zwei Fällen ist das Product eine Gerade. Diese Gerade kann dann entweder mit einem Punct oder einer Ebene zusammentreten. Dies giebt, wenn man in jedem dieser Fälle nur die 3 Stufenzahlen nebeneinander schreibt, folgende 4 Schemata:

$$
111,113,331,333 .
$$

In den Fällen c) und $d$ ) soll eine Gerade mit einem Punct oder einer Ebene zusammentreten. Da die Gerade aber wieder nur als Product zweier Puncte oder zweier Ebenen entstanden sein kann, so erhält man hier dieselben 4 Schemata. Es werde in jedem Schema das erste der 3 Elemente mit a oder $\alpha$, das zweite mit $b$ oder $\beta$, das dritte mit $c$ oder $\gamma$ bezeichnet, so erhält man die den 4 Schematen entsprechenden Producte:

die wir nach der Reihe mit

$$
a b c, a b \gamma, \alpha \beta c, \alpha \beta \gamma,
$$

$$
\rho, r, \sigma, s
$$

bezeichnen wollen, indem das erste und dritte eine Ebene, das zweita und vierte einen Punct dạrstellt. 
Ich will jetzt zunächst annehmen, dafs sowohl $a, b, c$, als auch $\alpha, \beta, \gamma$ variabel sind, und dafs jedes dieser Elemente dadurch entstanden sei, dâfs ein variabler Punct, oder eine variable Ebene, mit einer Reihe fester Elemente multiplicirt wurde. Eben so will ich annehmen, dafs die entstandenen Producte $(\varrho, r, \sigma, s)$ späterhin noch mit Reihen fester Elemente multiplicirt werden sollen. Hierdurch ist Alles auf die Betrachtung des vorigen Paragraphs reducirt.

Zuerst betrachte man das Product $\varrho \equiv a b c$. Hier können nach (\$.4) $a, b$ und $c$ als die letzten Ecken dreier lineal beweglicher offener Figuren angesehen werden; die Lage der Endstrablen der 3 offenen Figuren ist willkürlich, nur dafs sie beziehlich dürch $a, b, c$ gehen mũssen. Die Ebene $\varrho$ endlich soll als solche angesehen werden, die hernach noch mit einer Reihe fester Elemente zu multipliciren ist. Dieser Multiplication wurde in (\$.4) eine offene Figur zu Grunde gelegt, deren Anfangsstrahl in der Ebene $\varrho$ beweglich ist. Also treten hier 4 offene Figuren hervor; und zwar gehen die Endstrahlen der ersten 3 offenen Figuren, einzeln genommen, durch die Puncte $a, b, c$, und der Anfangstrahl der vierten liegt in der Ebene $\varrho \equiv a b c$. Da die Lage jener Endstrahlen im Übrigen willkürlich ist, so kann man sie leicht so annehmen, dafs die 4 in Betracht kommenden Grenzstrahlen paarweise zusammenfallen. Es sei $p$ ein in $a b$ beweglicher Punct, so läfst sich die Gerade $a b$ als der gemeinschaftliche Endstrahl der beiden ersten offenen Figuren, und die Gerade $\boldsymbol{p c}$ als der Endstrahl der dritten und der Anfangsstrahl der vierten setzen. Denn durch diese Annahmen werden die Bedingungen erfüllt, dafs die Endstrahlen beziehlich durch $a, b, c$ gehen und der Anfangsstrahl in der Ebene abc beweglich sein soll. Die eigenthümliche Lage der 4 Grenzstrablen in dem Producte $a b c$ ist also die, dafs sie parweise zusammenfallen und das eine Paar mit dem andern vereinigt liegt, $d . h$. von ihm geschnitten wird.

Betrachtet man zueitens das Product $r \equiv a b \gamma$, so sind hier $a$ und $b$ als letzte Ecken zweier offener Figuren anzusehen. Die Endstrahlen derselben, welche beziehlich durch $a$ und $b$ gehen müssen, im Übrigen aber willkürlich sind, kann man wieder zusammenfallen lassen, d. h. $a b$ als den gemeinschaftlichen Endstrahl derselben setzen. Die Ebene $\gamma$ ist nach (\$. 4.) als geometrischer Ort der letzten Seite einer offenen Figur zu betrachten. Der Endpunct dieser offenen Figur ist im Übrigen willkürlich; nur dafs er in der letzten Seite, also hier in der Ebene $\gamma$ liegen mufs. Der Punct $r \equiv a b y$, d. h. der Punct, in welchem die Gerade $a b$ die Ebene $\gamma$ schneidet, wird, 
wenn $r$ noch mit einer Reihe fester Elemente multiplicirt werden soll, zum Anfangspuncte einer vierten offenen Figur. Da der Endpunct der dritten in $\gamma$ willkürlich war, so kann man ihn mit dem Anfangspunct $(\boldsymbol{r})$ der vierten zusammenfallen lassen. Also fallen in diesem Falle zwei Grenzstrahlen $(a b)$ zusammen, und eben so zwei Grenzpuncte $(r)$, und diese liegen mit jenen vereinigt. In den beiden bisher betrachteten Fällen fallen also die 4 in Betracht kommenden Grenz-Elemente paarweise zusammen, und das eine Paar liegt mit dem andern vereinigt. Der Unterschied ist nur der, dafs im ersten Falle alle 4 Grenz-Elemente, im zweiten Falle zwei derselben Strahlen sind, die beiden andern Puncle.

Es werde jetzt das dritte Product $\sigma \equiv \alpha \beta c$ betrachtet. Hier ist nach (\$. 4.) die Ebene $\alpha$ als der geometrische Ort der'letzten Seite einer offenen Figur anzusehen, deren Endpunct willkürlich in dieser Seite, also auch willkürlich in der Ebene $\alpha$ liegt. Eben so ist $\beta$ als der geometrische Ort der letzten Seite einer offenen Figur zu betrachten, deren Endpunct willkürlich in $\beta$ angenommen werden kann. Man kann daher einen in der Kante $\alpha \beta$ beweglichen Punct $\boldsymbol{p}$ als gemeinschaftlichen Endpunct jener beiden offenen Figuren setzen. Ferner ist $c$ als die letzle Ecke einer offenen Figur zu betrachten, deren Endstrahl willkürlich durch $c$ geht. Wir setzen $p c$ als diesen Endstrahl. Endlich die Ebene $\sigma \equiv \alpha \beta c$, d. h. die Ebene, welche durch den Punct $c$ und die Kante $\alpha \beta$ gelegt ist, wird, wenn sie noch mit einer Reihe fester Elemente multiplicirt werden soll, zu dem geometrischen Orte des Anfangsstrahls einer vierten Figur. Da $p$ in $\alpha \beta$ beweglich ist, so ist $\sigma$ der geometrische Ort von $c p$; man kann also $c p$ als Anfangsstrahl der vierten offenen Figur setzen. Es sind demnach, wie im vorhergehenden Falle, zwei der GrenzElemente Strahlen, und die beiden andern sind Puncte; jene Grenzstrablen (cp) fallen zusammen; eben so diese Grenzpuncte $(p)$, und diese liegen mit jenen vereinigt. Der Unterschied zwischen diesem und dem vorhergehenden Falle ist nur der, dafs das Anfangs-Element der vierten Figur dort ein Punct, hier ein Strahl ist.

Das vierte Product endlich war $s \equiv \alpha \beta \gamma$. Hier ist jede der Ebenen $\alpha, \beta, \gamma$ als geometrischer Ort der Endseite einer offenen Figur zu betrachten, deren Endpunct also in jener Ebene willkürlich angenommen werden kann. Man kann daher den Durchschnittspunct (s) der 3 Ebenen $\alpha, \beta, \gamma$ als gemeinschaftlichen Endpunct der 3 offenen Figuren setzen; zugleich ist dieser 
Punct Anfangspunct der vierten. Also sind dann alle 4 Grenz-Elemente Puncte, welche in einem Punct $s$ zusammenfallen. Auch in diesem Falle kann man sagen, dafs die 4 Grenz-Elemente paarweise zusammenfallen und das eine Paar mit dem andern vereinigt liegt. Dies ist also das Gemeinschaftliche in allen vier Fällen. Im ersten Falle sind alle Grenz-Elemente Strahlen, im letzten, Puncte; in den beiden mitlleren Fällen sind zwei Grenz-Elemente Strahlen, die beiden andern Puncte; und zwar ist das Anfangs-Element der vierten Figur im zweiten Falle ein Punct, im dritten ein Strahl.

Es wurde oben angenommen, dafs $a, b, c, \alpha, \beta, \gamma$, dadurch entstanden seien, dafs ein variabler Punct, oder eine variable Ebene, mit einer Reihe fester Elemente multiplicirt sei; wobei es gleichgültig ist, ob diese Reihe aus einem, oder aus mehren Elementen besteht. Das erlangte Resultat bleibt indessen bestehen, auch wenn jene Bedingung nicht erfüllt wird. In der That ist z. B. a zwar variabel, aber nicht durch Multiplication eines beweglichen Elements mit einem oder mehreren festen Elementen entstanden: also können wir dennoch $a$ als den Anfangspunct einer offenen Figur setzen, an den sich aber sogleich der Endstrahl derselben anschliefst; und die oben gezogenen Folgerungen bleiben bestehen. Durch das Wegfallen der constanten Factoren ist nur das Wegfallen der Ecken und Seiten der offenen Figur bedingt, und diese besteht blofs aus den beiden Grenz-Elementen. Eben so, wenn die Ebene $\alpha$ zwar variabel ist, aber nicht durch Multiplication eines beweglichen Elements mit einem oder mehreren festen Elementen entstanden ist, kann man dennoch $\alpha$ als den geometrischen Ort des Anfangsstrahls einer offenen Figur setzen, an welchen sich aber sogleich der Endpunct derselben anschliefst. Die offene Figur besteht dann wiederum nur aus den beiden Grenz-Elementen; in den übrigen Folgerungen wird nichts geändert. Ganz auf dieselbe Weise kann man die Annahme, dafs die entstehenden Producte noch hernach mit einer Reihe fester Elemente multiplicirt werden sollen, ganz wegfallen lassen.

Ferner wurde oben angenommen, dafs in jedem der 4 Producte alle 3 Factoren variabel sind. Sind alle 3 constant, so ist auch ihr Product constant und kann also ohne $W$ eiteres als eins der festen Elemente gesetzt werden. Sind 2 derselben constant, so ist dann das variable Element entweder fortschreitend mit 2 festen Elementen multiplicirt, oder mit deren Product, d. h. mit Einem festen Element. In beiden Fällen setzt sich die an das variable Element sich anschliefsende offene Figur nur fort. Es bleibt also nur der Fall zu betrachten, wo eins der 3 Elemente fest ist, die beiden andern beweglich sind. 
Dieser Fall erfordert um so mehr Beachtung, da er bei der Erzeugung der Oberflächen bei weitem der häufigste ist. Angenommen also, es sei etwa der Punct $a$, den wir bisher als beweglich setzten, constant, so tritt kein anderer Unterschied hervor, als dafs der durch a gehende Strahl, welcher bisher als Endstrahl einer offenen Figur sich zeigte, jetzt durch den festen Punct $a$ geht, oder, anders ausgedrückt, dafs die Ecke $a$, welche bisher beweglich war, jetzt fest wird. In dem vorher gefundenen Resultate wird im Übrigen nichts geändert. Ja auch der Wort-Ausdruck desselben kann unverändert bleiben, wenn man den festen Punct $a$, nebst dem von ihm ausgehenden Strahle, gleichfalls als offene Figur setzt; und zwar den Strahl als Endstrabl derselben. Hierbei ist es gleichgültig, ob man den festen Punct $a$ als Anfangspunct der offenen Figur setzt, oder als eine Ecke derselben, indem man dieser noch beliebige feste Seiten und Ecken vorangeben läfst. Immer kann man die ganze Figur, mit Ausnahme des durch $a$ gehenden willkürlichen Endstrahles, als unbeweglich annehmen. Wir nennen eine solche offene Figur, da sie von dem veränderlichen Elemente unabhängig ist, zum Unterschiede von den früher betrachteten, eine unabhüngige offene Figur. Ist zweitens eine der Ebenen, etwa $\alpha$, constant, so wird der in $\alpha$ liegende Punct, welcher bisher als Endpunct einer offenen Figur sich zeigte, jetzt ein in der festen Ebene $\alpha$ liegender Punct. Man wird daher auch diesen Punct als Endpunct einer offenen Figur setzen können; indem man eine solche offene Figur, deren Endpunct in einer festen Ebene liegt, gleichfalls unabhängig nennt.

Von hier aus gelangt man sogleich zur Construction eines beliebigen Products, welches das bewegliche Element $x$ beliebig oft als Factor enthält, indem man den Begriff der Verkettung offener Figuren, wie er von mir (S. dieses Journal Bd. 42. S. 190) der Erzeugung ebener Curven zum Grunde gelegt ist, nach Anleitung der so eben gegebenen Entwickelung auf den Raum überträgt. Wenn man nämlich einen beweglichen Punct $x$ zum Anfangspuncte mehrerer offener Figuren macht, dann aus dreien derselben, oder aus zweien und einer unabhängigen offenen Figur, eine neue offene Figur in der Art bildet, dafs die 4 Grenz-Elemente (nàmlich die 3 End-Elemente der 3 ersteren und das Anfangs-Element der neuen) paarweise zusammenfallen, während zugleich das eine Paar mit dem andern vereinigt liegt, und dann fortfährt, die jedesmal noch übrigen offenen Figuren auf die angegebene Art zusammenzuschliefsen, bis sich zuletzt alle variablen offenen Figuren zu einer einzigen vereinigt haben: so nenne ich das ganze System dieser offenen 
Figuren eine Verkettung derselben; und zwar eine Verkettung nten Grades, wenn $n$ offene Figuren von dem Anfangs-Element der Verkettung ausgehen. Wir sagen ferner, dafs eine Verkettung offener Figuren im Raume sich lineal bewege, wenn alle abhängigen offenen Figuren, - aus denen sie besteht, sich lineal bewegen. Mittels dieser Begriffe läfst sich nun das Resultat dieses Paragraphs in dem folgenden Satze aussprechen:

„Jedes Product, welches $\boldsymbol{n}$ mal den variablen Punct $\boldsymbol{x}$ als Factor enthält, und welches von erster oder dritter Stufe ist, aber keinen Factor nullter Stufe einschliefst, läfst sich durch eine lineal bewegte Verkettung $n$ ten Grades in der Art darstellen, dafs für jeden Punct $\boldsymbol{x}$ das Product, entweder der letzten Ecke der Verkettung, oder der Ebene, in welcher die letzte Seite derselben beweglich ist, congruent.sei. Und umgekehrt läfst sich die letzte Ecke oder die Ebene der letzten Seite jeder Verkettung nten Grades, durch ein solches Product darstellen.

\section{S. 6 .}

Erzeugung der algebraischen Oberflächen durch lineale Bewegung geschlossener Verkettungen.

Es werde jetzt endlich ein beliebiges Product nullter Stufe betrachtet, welches $n$ mal $x$ als Factor enthält, aber keinen Factor nullter Stufe einschliefst. Ich habe in dem vorhergehenden Aufsatze gezeigt, dafs man in einem solchen Producte jeden Factor, also auch $x$, ohne Klammern nach der letzten Stelle bringen kann. Dann erhält das Product die Form $\widetilde{\omega} x$, wo $\widetilde{w}$ ein Product dritter Stufe ist, welches $(n-1)$ mal den Punct $x$ als Factor enthält. Hat man nun die Gleichung

$$
\widetilde{\omega} x=0,
$$

so drückt sie aus, dafs der Punct $x$ in der Ebene $\widetilde{w}$ liegt. Die Ebene $\widetilde{\omega}$ aber läfst sich nach dem vorigen Paragraph als die Ebene darstellen, in welcher die Endseite einer Verkettung $(n-1)$ ten Grades beweglich ist. Also drückt die Gleichung $\widetilde{\omega} x=0$ die Möglichkeit aus, jene Seite durch den Punct $x$ zu legen, oder, anders ausgedrückt, die Möglichkeit, das End-Element der Verkettung mit dem Anfangs-Element derselben zusammenfallen zu lassen. Wir nennen eine solche Verkettung, deren End-Element mit ihrem AnfangsElement zusammenfällt, eine geschlossene Verkettung; und zwar nten Grades, wenn $n$ offene Figuren von dem Anfangs-Elemente $(x)$ ausgehen (die letzte, 
welche $x$ zum End-Elemente hat, eingerechnet). Dann verwandelt sich der Salz 5. der Einleitung in folgenden Satz:

„Der Anfangspunct einer sich lineal bewegenden geschlossenen Verkettung $n$ ten Grades beschreibt eine Oberfläche nter Ordnung,"

und umgekehrt:

„Jede algebraische Oberfläche läfst sich als Ort einer sich lineal bewegenden geschlossenen Verkettung darstellen."

Stellin, im Juli 1852. 